\title{
EVALUACIÓN DEL MÉTODO DE IGNICIÓN PARA DETERMINAR MATERIA ORGÁNICA EN SUELOS DE LA PROVINCIA EL ORO-ECUADOR
}

\author{
Barrezueta-Unda, S. $^{1}$; Cervantes-Alava, A. ${ }^{1}$; Ullauri-Espinoza, M. ${ }^{1}$;

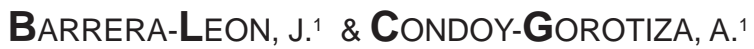

\begin{abstract}
RESUMEN
El método de pérdida de peso por ignición (Loss On Ignition -LOI) se utiliza para estimar la materia orgánica del suelo (MOS) pero tiene sus ventajas y limitaciones, frente a otros métodos que lo calculan partiendo del carbono orgánico (CO). El objetivo del trabajo fue, calibrar el método LOI para utilizarlo en suelos de origen aluvial cultivados con cacao (Theobroma cacao L.). Se utilizaron 30 muestras de suelo extraída entre 0 a $30 \mathrm{~cm}$ de profundidad, en las que se determinaron la MOS por LOI a 350,430 y $600^{\circ} \mathrm{C}$ durante $2 \mathrm{~h}$ y que fueron contrastadas con las determinaciones del por CO combustión seca (DC) y Walkley y Black (WB). Los valores obtenidos por LOI aumentaron de $3,46 \%<4,95 \%<7,66 \%$, al incrementar la temperatura, siendo inferiores a los obtenidos con DC $(1,85 \%)$ y WB $(1,96 \%)$. El mejor ajuste lineal obtenido entre LOI y DC fue, a $430^{\circ} \mathrm{C}$ con un coeficiente de explicación (R2) igual a 0,83 . Aunque el método LOI a $430^{\circ} \mathrm{C}$ fue el de mejor ajuste para ser utilizada en el cálculo de la MOS se deben realizar más repeticiones para un mejor modelo de calibración.
\end{abstract}

Palabras clave: combustión seca, Método de Walkley-Black, Theobroma cacao L.

\begin{abstract}
Assessing Loss on ignition to determine organic matter in soils of the El Oro province of Ecuador.

The Loss On Ignition (LOI) method is used to estimate the soil organic matter (SOM) but has its advantages and limitations compared to other methods that calculate it from organic carbon (OC). The objective of the work was to calibrate the LOI method for use in alluvial soils cultivated

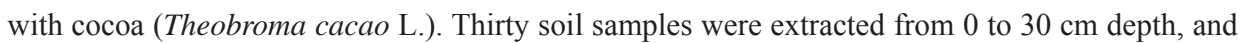

1.- Universidad Técnica de Machala, Facultad de Ciencias Agropecuarias, Av. Panamericana km 5 1/2, 070222 ; Machala, Ecuador. Email: sabarrezueta@utmachala.edu.ec

Manuscrito recibido el 8 de abril de 2020 y aceptado para su publicación el 24 de agosto de 2020.

Barrezueta-Unda, S.; Cervantes-Alava, A.; Ullauri-Espinoza, M.; Barrera Leon, J.; Condoy-Gorotiza, A. Evaluación del método de ignición para determinar materia orgánica en suelos de la provincia el Oro-Ecuador. FAVE - Ciencias Agrarias 19 (2): 25-36. CC BY-NC-SA 4.0 


\section{S. Barrezueta-Unda et al.}

the following were determined SOM by LOI at 350,430 and $600^{\circ} \mathrm{C}$ for $2 \mathrm{~h}$. These samples were contrasted with the dry combustion (DC) and Walkley and Black (WB) methods for OC determinations. The values obtained by LOI increased from $3,46 \%<4,95 \%<7,66 \%$, when the temperature increased, being lower than those obtained with DC $(1,85 \%)$ and WB $(1,96 \%)$. The best linear adjustment obtained between LOI and DC was, at $430^{\circ} \mathrm{C}$ with an explanation coefficient (R2) equal to 0.83 . Although the $\mathrm{LOI}$ method at $430^{\circ} \mathrm{C}$ was the best fit to be used in the SOM calculation, more repetitions should be done for a better calibration model.

Key words: efdry combustion, Walkley-Black method, Theobroma cocoa L.

\section{INTRODUCCIÓN}

El contenido de materia orgánica de los suelos (MOS) es una de las propiedades más importantes debido a que desempeña un papel clave en varios procesos del ecosistema. Entre los cuales se pueden citar: la formación de la estructura del suelo, la retención de agua, la desintoxicación de sustancias químicas antropogénicas, el secuestro de carbono, el ciclo de los nutrientes y el suministro de energía a los microorganismos del suelo (Martínez et al., 2018; Zhang et al., 2005). Por lo tanto, es importante desarrollar métodos apropiados para cuantificar los contenidos de la materia orgánica del suelo (Hoogsteen et al., 2015).

La mayoría de las técnicas tradicionales utilizadas para el análisis del suelo consumen mucho tiempo y generan residuos que en numerosos de los casos causan efectos adversos para el medio ambiente (Salehi et al., 2011; J. Wang et al., 2013). Así, el método propuesto por Walkley y Black (1934) es el más utilizado en todo el planeta para determinar los niveles de MOS, consiste en la oxidación húmeda de la muestra de suelo con dicromato de potasio en una solución ácida. Esto genera residuos que deben ser gestionados adecuadamente (Pribyl, 2010; Sato et al., 2014). Algunos autores expresan que el método Walkley y Black (WB) es una oxidación incompleta del carbono orgánico (CO), lo que implica que se necesita un factor de corrección (FQ), que varía de un suelo a otro, y depende en gran medida del tamaño de la muestra y el tipo de reactivo utilizado (Hoogsteen et al., 2018; Howard \& Howard, 1990; Pribyl, 2010). El FQ de uso común en la mayoría de los laboratorios de suelos es 1,724 también llamada factor Van Bemmelen (1890); este factor se basa en el supuesto de que la MOS contiene $58 \%$ de carbono (Pribyl, 2010). Sin embargo, Kamara et al., (2007) y Pribyl, (2010) recomiendan utilizar el FQ de acuerdo con el horizonte y el tipo de cultivo, y puede oscilar entre 0,5 y 3,18.

Otro método analítico para determinar la MOS es la combustión seca (DC), basada en el principio de combustión de Dumas que estima el carbono total (Vitti et al., 2016), que se presenta como el método más preciso, pero requiere un analizador elemental calibrado con estándares de suelo certificados (Hoogsteen et al., 2018; Martínez et al., 2018). El método de DC mide directamente el carbono total del suelo sin necesidad de un factor de corrección (Salehi et al., 2011). En este método, la muestra se calcina entre $950-1300^{\circ} \mathrm{C}$, obteniendo $\mathrm{CO} 2$ que pasa a través de un catalizador que mide, mediante espectroscopía de absorción infrarroja, el carbono total (Dias et al., 2013; Pribyl, 2010). 
Otra opción para determinar la MOS es la aplicación del método de calcinación o muflado que consiste en la medición de la pérdida de peso por ignición (Loss On Ignition, LOI) de la muestra de suelo. En este método los investigadores proponen la calcinación de diferentes cantidades de muestras de suelo que van de 2 a $100 \mathrm{~g}$, de temperaturas entre 220 a $600^{\circ} \mathrm{C}$ y tiempos que oscilan de 2 a $48 \mathrm{~h}$ en un horno de mufla (Dias et al., 2013; Kamara et al., 2007; Martínez et al., 2018; Westman et al., 2006). El objetivo del método LOI, es destruir la MOS por calor ocasionado la oxidación de las formas condensadas de humus y residuos orgánicos apenas alterados. Sin embargo, no es recomendable en suelos con presencia de carbonatos, porque la combustión llevaría a una sobreestimación de la MOS (Westman et al., 2006).
El objetivo del presente estudio fue calibrar el método LOI utilizando tres temperaturas de calcinación para utilizarlo en suelos de origen aluvial cultivados con cacao (Theobroma cacao L.).

\section{MATERIALES Y MÉTODOS}

\section{Descripción de las muestras}

El muestreo se realizó en campos cultivados con cacao al noroeste de la provincia de El Oro, Ecuador entre las coordenadas $3^{\circ} 6^{\prime} 17,50^{\prime \prime}$ y $3^{\circ} 28^{\prime} 37,60^{\prime}$ ' S y $79^{\circ} 44^{\prime} 25^{\prime}, 75^{\prime \prime}$ y 79॰55'10,90” O (Figura 1). El rango anual de precipitación en la zona de estudio se ubica entre 500-1700 $\mathrm{mm}$, y la temperatura promedio es de $26^{\circ} \mathrm{C}$.

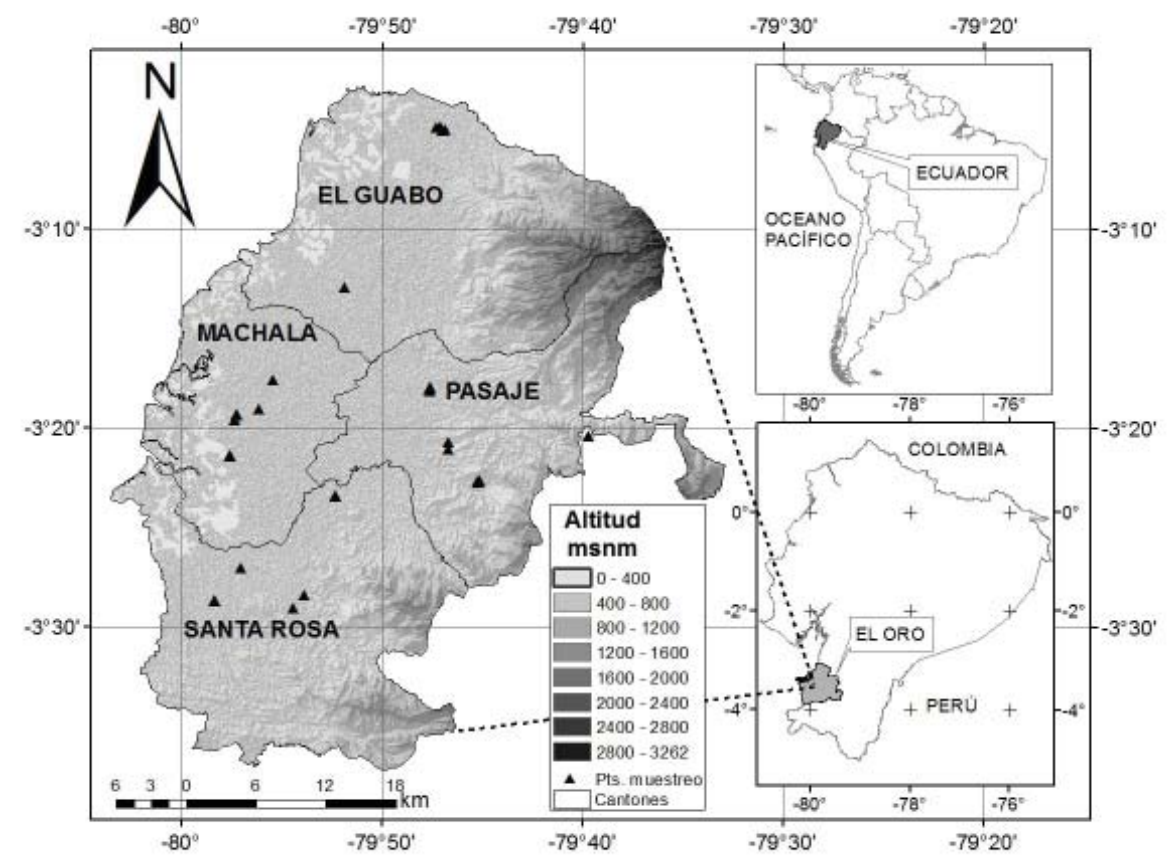

Figura 1. Ubicación de los puntos de muestreo en la provincia de El Oro, Ecuador.

Figure 1. Location of sampling points in the province of El Oro, Ecuador.

Revista FAVE - Ciencias Agrarias 19 (2) 2020 | 
El origen geológico de esos suelos es aluvial, y están formados por sedimentos fluviales y marinos (Moreno et al., 2016).

Durante el 2015, se reunió un conjunto de 30 muestras de suelo tomadas de cero a $30 \mathrm{~cm}$ de profundidad. Las muestras de suelo fueron secadas aire durante varios días, luego trituradas y pasadas por un tamiz de malla $2 \mathrm{~mm}$ y luego se realizaron diferentes determinaciones en laboratorio a fin de estimar la textura (método pipeta), $\mathrm{pH}$ (relación 1:2,5 suelo-agua), capacidad de intercambio catiónico (CIC) y concentración de $\mathrm{Ca}, \mathrm{Cu}, \mathrm{Fe}$ y $\mathrm{Mg}$ (Tabla 1).

\section{Método Walkley y Black (WB) o digestión húmeda}

La primera determinación del CO se realizó mediante el método WB, que se llevó a cabo en el Laboratorio de Suelos del Instituto Nacional de Investigaciones Agrícolas del Ecuador. Consistió en pesar $1 \mathrm{~g}$ de suelo que se colocó en un matraz Erlenmeyer de $500 \mathrm{ml}$, se añadieron $10 \mathrm{ml}$ de $\mathrm{K} 2 \mathrm{Cr} 2 \mathrm{O} 7 \mathrm{y}$ $10 \mathrm{ml}$ de ácido sulfúrico (H2SO4 al 96\%), se agitó durante 30 segundos y se dejó en reposo hasta que se enfrió (Schulte, 1987). A continuación, se añadieron $100 \mathrm{ml}$ de agua destilada y $1 \mathrm{ml}$ de difenilamina y $5 \mathrm{ml}$ de ácido fosfórico (H3PO4 al 85\%). Luego, se tituló con la adición sulfato de hierro (Fe2SO4), hasta alcanzar un punto final de color verde y se anotó su consumo (Pribyl, 2010). Para obtener la MOS se multiplico por el FQ 1,724.

\section{Método de combustión seca \\ (Dry Combustion-DC)}

La determinación del $\mathrm{CO}$ por el método de DC fue realizada en el Servicio de Apoyo a la Investigación de la Universidad de La Coruña (España), a través de un analizador elemental modelo Flash EA1112 (Thermo Finnigan, Colorado, EE. UU.), donde las muestras de suelo se encapsularon en estaño y se colocaron en una columna de cromatografía para ser calcinadas a $1115^{\circ} \mathrm{C}$.

\section{Método pérdida por ignición} (LOI) o gravimétrico

En los 30 sitios muestreados se tomó un segundo grupo de suelos de aproximadamente $500 \mathrm{~g}$ por muestra que fueron almacenados en recipientes de plástico en el laboratorio de suelos de la Universidad Técnica de Machala (UTMACH), y que fue el material con el que se realizó la metodología de LOI durante el año 2018.

Para determinar la MOS por el método LOI o gravimétrico, las muestras se secaron al horno a $105^{\circ} \mathrm{C}$ durante $3 \mathrm{~h}$, se enfriaron en una desecadora y se pesaron antes de ser calcinadas a 350,430 y $600{ }^{\circ} \mathrm{C}$ durante $2 \mathrm{~h}$ en un horno de mufla (Nabertherm, Lilienthal, Alemania). Después de la combustión, las muestras se enfriaron en un desecador y se volvieron a pesar. Con estos valores, se calculó el porcentaje de MOS mediante la siguiente ecuación (1) propuesta por Schulte y Hopkins (1996). Todo el procedimiento se repitió por triplicado y fue realizado en el laboratorio de suelos de la Universidad Técnica de Machala (Ecuador). 


$$
L O I=\left(\frac{\text { Peso seco aire del suelo }- \text { peso de la muestra después de la ignición }}{\text { Peso seco al aire del suelo }}\right) \times 100
$$

En este método se asume que la pérdi- los métodos WB y LOI; para la comparada de peso de la muestra por ignición se ción de las medias se aplicó la prueba de debe únicamente a la pérdida de materia Tukey $(\mathrm{P}<0,05)$. orgánica, producto de la siguiente reacción: C6H12O6 (materia orgánica) $+6 \mathrm{O} 2+$ calor $\rightarrow 6 \mathrm{CO} 2+6 \mathrm{H} 2 \mathrm{O}$. (Bianchi et al., 2008).

\section{Análisis estadístico}

Se aplicó la estadística descriptiva a todos los resultados obtenidos en la investigación. La ecuación de regresión lineal se calculó para estimar el carbono orgánico, a partir de los valores de la MOS (variable independiente) y de $\mathrm{CO}$ (variable dependiente), obtenido por el método DC. También se realizó, el análisis de la varianza (ANOVA) de un factor con los valores obtenidos de
Para la validación del modelo obtenido se calcularon el coeficiente de determinación (R2) y el error cuadrático medio (Root Mean Square Error-RMSE) con la ecuación (2).

$$
R M S E=\sqrt{\sum_{i=1}^{n} \frac{\left(P_{x i}-M_{x i}\right)^{2}}{n}}
$$

Donde Pxi es la estimación de MOS por el método de LOI en la ubicación xi, Mxi es el COS medido por el método DC en la ubicación xi y n es el número de muestras.

Todo el proceso estadístico fue realizado utilizando el software SPSS versión 23 (SPSS, 2013).

Tabla 1. Estadísticas descriptivas de algunas propiedades del suelo.

Table 1. Descriptive statistics of some soil properties1 Capacidad de Intercambio Catiónico (CIC).

\begin{tabular}{|l|c|c|c|}
\hline & Media & Mínimo & Máximo \\
\hline Arena (\%) & $38,32 \pm 13,99$ & 16,00 & 72,00 \\
\hline Limo (\%) & $34,02 \pm 11,69$ & 14,70 & 61,40 \\
\hline Arcilla (\%) & $27,66 \pm 11,19$ & 9,30 & 52,60 \\
\hline $\mathrm{pH}$ & $6,90 \pm 0,93$ & 4,86 & 8,57 \\
\hline $\mathrm{ClC}\left(\mathrm{cmol}(+) \mathrm{kg}^{-1}\right)$ & $30,51 \pm 12,30$ & 6,60 & 52,20 \\
\hline $\mathrm{Ca}\left(\mathrm{cmol}(+) \mathrm{kg}^{-1}\right)$ & $26,96 \pm 13,10$ & 5,70 & 45,80 \\
\hline $\mathrm{Cu}(\mathrm{mg} / \mathrm{kg})$ & $11,03 \pm 5,74$ & 2,90 & 24,50 \\
\hline $\mathrm{Fe}(\mathrm{mg} / \mathrm{kg})$ & $205,43 \pm 230,97$ & 47,00 & 1269,00 \\
\hline $\mathrm{Mg}\left(\mathrm{cmol}(+) \mathrm{kg}^{-1}\right)$ & $4,10 \pm 2,35$ & 1,20 & 13,90 \\
\hline
\end{tabular}

Fuente: (Barrezueta-Unda, 2019) 


\section{RESULTADOS Y DISCUSIÓN}

Los niveles determinados de $\mathrm{CO}$ con los métodos DC y WB oscilaron entre bajo y medio (Fassbender y Bornemisza, 1987), siendo ligeramente inferior los valores estimados por DC (media: 1,85\% $\pm 1,08$; rango:0,49 a $4,80 \%$ ), con respecto a los obtenidos por el método WB (media: $1,96 \% \pm 1,08$; rango: $0,75 \%$ a $4,87 \%$ ). Investigaciones llevadas a cabo por Díaz Zorita (1999) y Mikhailova et al. (2003), muestran lo contrario, valores de COS obtenidos por DC superiores a los determinados por WB en suelos con manejo agrícola en Argentina (Typic Hapludoll) y Rusia (Typic Hapludoll). Igual tendencia observaron Richardson y Bigler (1982) en suelos calcáreos en Estados Unidos. El coeficiente de variación fue alto $(\mathrm{DC}=54,59 \%$; $\mathrm{WB}=55,10 \%$ ), producto de heterogeneidad del $\mathrm{CO}$ en los suelos cultivados con cacao, lo cual es normal (Snoeck et al., 2016; van Vliet et al., 2015), debido al continuo flujo de la hojarasca y ramas al suelo, que en algunos casos se sitúan entre 5 y 21 t ha- 1 año (Pérez-Flores et al., 2017). Este factor afecta el grado de homogeneidad de los compuestos fenólicos y de lignina e influyen en la oxidación total de carbono, en la disolución de $\mathrm{H} 2 \mathrm{SO} 4$ cuando se aplica el método WB, produciendo una sobre estimación del CO (Pribyl, 2010).

Los valores medios de MOS obtenidos con el método de WB $(3,38 \% \pm 1,86)$ fueron significativamente diferentes $(\mathrm{p}<0,05)$ a los obtenidos con el método LOI independientemente de la temperatura de calcinación empleada (tabla 2). Se obtuvo un valor mayor de MOS con LOI600 (8,47\% $\pm 3,34)$ que con LOI350 $(4,91 \% \pm 1,84)$ y LOI430 $(4,99 \% \pm 2,07)$, los cuales, a su vez, no difirieron entre sí. Resultados que se relacionan con la pérdida de agua estructural en las arcillas a temperaturas $>550^{\circ} \mathrm{C}$ (Boyle, 2004; Dias et al., 2013; Westman et al., 2006). Mientras, entre 300 y $500^{\circ} \mathrm{C}$ se produce la oxidación de compuestos orgánicos (proteínas, aminoácidos, hidratos de carbono, ácidos orgánicos, ce-

Tabla 2.- Estadística descriptiva y prueba de Tukey $(p<0.05)$ para la materia orgánica del suelo obtenida por los métodos: Walkley-Black (WB) y Pérdida de peso por ignición (LOI).

Table 2.- Descriptive statistics and Tukey test $(p<0.05)$ for soil organic matter obtained by the Walkley-Black (WB) and Loss on Ignition (LOI) methods.

\begin{tabular}{|l|r|c|r|r|}
\hline Métodos & Media & $\begin{array}{c}\text { Coeficiente } \\
\text { de variación } \\
(\%)\end{array}$ & Mínimo & Máximo \\
\hline WB $^{1}$ & $3,38 \pm 1,86 \mathrm{a}$ & 55,03 & 1,30 & 8,40 \\
\hline LOI350 & $4,91 \pm 1,84 \mathrm{~b}$ & 37,48 & 1,61 & 9,19 \\
\hline LOI430 & $4,99 \pm 2,07 \mathrm{~b}$ & 41,48 & 1,36 & 10,65 \\
\hline LOI600 & $8,47 \pm 3,34 \mathrm{c}$ & 39,43 & 2,25 & 17,41 \\
\hline
\end{tabular}

Diferentes letras indican la significación estadística $(p<0,05)$

Walkley y Black (BW) con factor de corrección 1,724 
ras, resinas, lignina, etc.) y de sustancias húmicas estables (Bianchi et al., 2008; Gelati et al., 2009; Morona, Melquíades y Muller, 2017). Schulte y Hopkins (1996) y Dias et al., (2013) expresan que el método LOI es insensible al factor del número de muestras colocadas en un horno de mufla, pero sí al peso de las muestras utilizadas. En este caso se utilizó igual peso para todos los tratamientos, con lo que se descarta que los valores obtenidos variaron por la técnica que se utilizó para la ignición de las muestras.

A partir de los análisis de regresión entre los valores de MOS obtenidos con WB y LOI sobre COS obtenido por DC (Figura 2 ), se observa que existe una fuerte relación entre $\mathrm{DC}$ y WB $(\mathrm{R} 2=0,96)$ que ajustó al modelo $\mathrm{y}=0,91 \mathrm{x}+0,06$, similar al determinado en suelos de clima tropical por Kamara et al., (2007) y Alves Fernandes et al., (2015) con un $\mathrm{R} 2=0,98(\mathrm{y}=1,58 \mathrm{x}+$ $0,23)$ y $R 2=0,95(y=4,37 x+0,83)$ respectivamente. Drover y Manner (1975) y Kamara et al. (2007), señalan que elevados coeficientes de regresión entre los métodos DC y WB se asocia a suelos no superficiales y provenientes de zona tropicales, aquí la MOS se oxida más fácilmente por la humedad ambiental (in situ), a la que están expuestos los suelos superficiales.

La regresión lineal entre el método LOI a $350^{\circ} \mathrm{C}$ y el $\mathrm{CO}$ obtenido por DC mostró una pendiente positiva significativamente distinta de 0 . Este resultado no se ajusta a las investigaciones de Salehi et al., (2011), que lograron un $\mathrm{R} 2 \geq 0,710$ con LOI durante dos horas entre $300-420^{\circ} \mathrm{C}$, en suelos ubicados en una zona semiárida de Irán. En cambio, en el tratamiento LOI430, el R2 fue de 0,84 , menor si se compara con los resultados de Howard y Howard (1990) que estimaron un $\mathrm{R} 2$ de 0,98 a $550{ }^{\circ} \mathrm{C}$ durante 3 horas, en suelos correspondiente a un uso de bajos en Inglaterra con niveles de $\operatorname{COS}>15 \%$, pH entre 3,3 a 6,4 y de bajo contenido de arcilla $(<21,0 \%)$; por otra parte, Alves Fernandes et al. (2015), en Brasil obtuvieron una baja correlación $(\mathrm{R} 2=0,630, \mathrm{n}=17)$ a $450^{\circ} \mathrm{C}$ de ignición durante 5 horas en Inceptisol, Spodosol e Histosol, pero en oxisoles a la misma temperatura y tiempo obtuvieron un $\mathrm{R} 2=0,850$ con, once muestras). Las muestras de suelo tratadas a $600{ }^{\circ} \mathrm{C}$ (LOI600), fueron las de menor coeficiente de determinación $(\mathrm{R} 2=140 ; \mathrm{y}=0,128 \mathrm{x}+0,874)$. Wang et al. (2012), expresan que se pueden alcanzar mejores ajustes para estimar COS a temperaturas entre 375 y $550{ }^{\circ} \mathrm{C}$ durante $12 \mathrm{~h}$, pero estos estudios se restringen a los suelos de regiones semiáridas de China solo en suelo de regiones semiáridas China. Aunque no se realizó pruebas para determinar si los suelos son calcáreos, la información recaba de los suelos ecuatoriano indican que no son del tipo calcáreo (Moreno et al., 2016), aunque algunos suelos en estudio tuvieron un $\mathrm{pH}$ mayor a 8 (Tabla 1), por el nivel alto de Ca el cual es aporte de la hojarasca del cacao (Hartemink, 2005).

Los valores predichos de CO y la validación realizada con la prueba RMSE (Tabla 3 ), no mostraron diferencias significativas entre los valores obtenidos por WB y LOI $(\mathrm{p}<0,05)$. Las medias variaron entre 1,76 $\%$ (WB) y $1,85 \%$ (LOI), con los altos CV para BW (52,60\%) y LOI430 (50,08\%), a pesar de haber obtenido ecuaciones de regresión con un alto valor del R2. Mientras que los valores extremos predichos por LOI600 fueron los más próximos $(1,16 \%$ y $2,83 \%)$, contrario a los determinados en los valores observados (Tabla 2), 
S. Barrezueta-Unda et al.

(a)

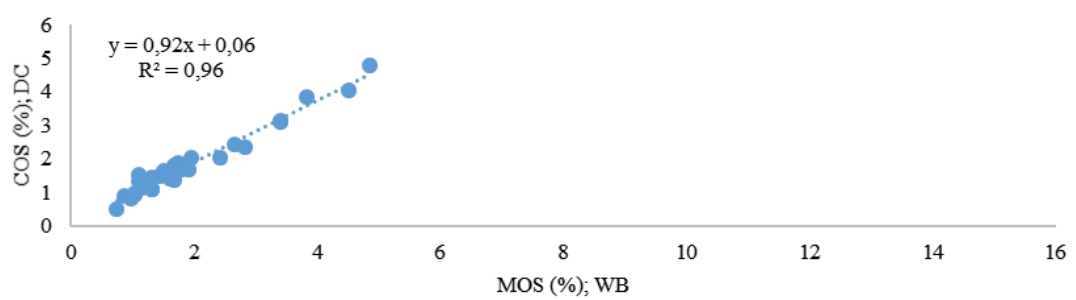

(b)

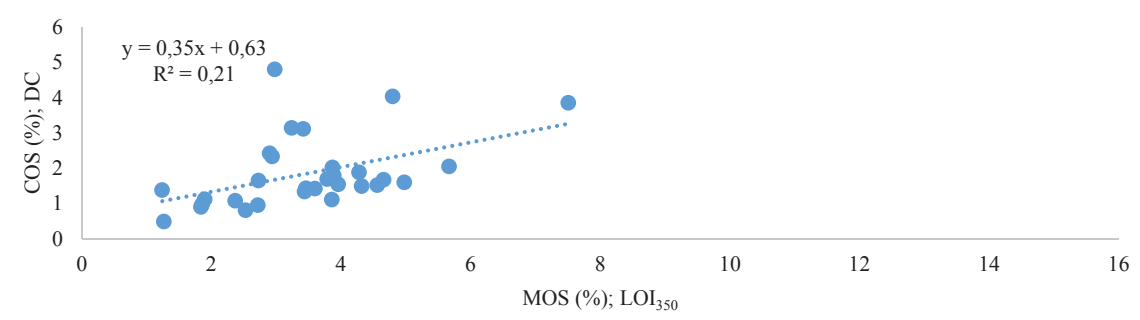

(c)

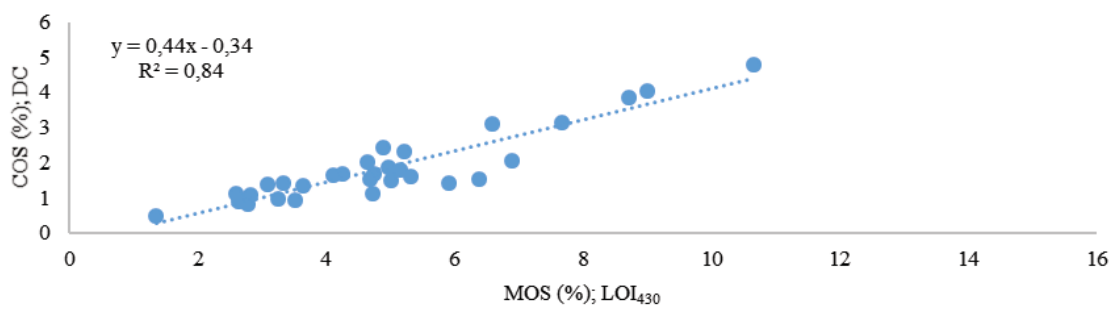

(d)

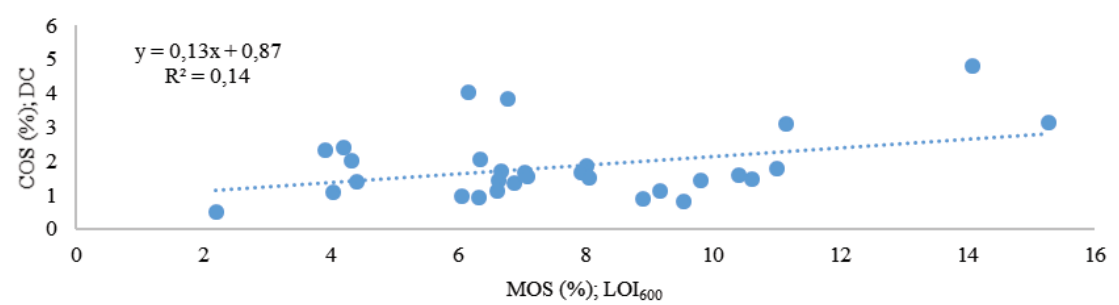

Figura 2.- Regresiones lineales entre la materia orgánica del suelo (MOS) determinada por los métodos Walkley-Black (WB) y Pérdida de peso por ignición (LOI) y el carbono orgánico del suelo (COS) obtenido por DC. (a) WB/DC; (b) LOI350/DC; (c) LOI430/DC; (d) LOI600/DC Figure 2.- Linear regressions between the soil organic matter (MOS) determined by the WalkleyBlack (WB) and Loss on Ignition (LOI) methods and the organic Carbon (COS) obtained by DC (a) WB/DC; (b) LOI350/DC; (c) LOI430/DC; (d) LOI600/DC 
Tabla 3. Prueba Tukey (5\%) y validación de valores predichos para obtener la materia orgánica del suelo.

Table 3. Tukey test (5\%) and validation of predicted values to obtain soil organic matter.

\begin{tabular}{|c|c|c|c|c|c|}
\hline Métodos & Media & $\begin{array}{c}\text { Coeficiente de } \\
\text { variación (\%) }\end{array}$ & Mínimo & Máximo & RMSE \\
\hline \multirow{2}{*}{$\begin{array}{c}\text { BW } \\
\text { LOI350 } \\
\text { LOI430 } \\
\text { LOI600 }\end{array}$} & $1,76 \pm 0,93 a$ & 52,60 & 0,51 & 4,47 & 0,14 \\
\cline { 2 - 6 } & $1,85 \pm 0,47 a$ & 25,22 & 1,07 & 3,26 & 0,97 \\
\cline { 2 - 6 } & $1,85 \pm 0,93 a$ & 50,08 & 0,26 & 4,38 & 0,35 \\
\hline
\end{tabular}

Diferentes letras indican diferencias significativas $(p<0,05)$

El mejor ajuste se obtuvo en BW con un RMSE de 0,14, seguido de LOI430 con 0,35 . Salehi et al., (2011) también encontraron una relación similar entre $500^{\circ} \mathrm{C}$ y $550^{\circ} \mathrm{C}$, donde el R2 disminuye y el RMSE es cercano a 1. Numerosos investigadores han sugerido que cuando la MOS es obtenida por el método LOI, las ecuaciones se desarrollan por separado para diferentes tipos de suelos y profundidades (Dias et al., 2013; Maranhão et al., 2017; Salehi et al., 2011).

\section{CONCLUSIONES}

Los valores de $\mathrm{CO}$ obtenidos por los métodos DC y WB tuvieron un R2 alto, lo que indica que existe un alto grado de fiabilidad de los resultados obtenidos por WB, aunque los niveles de Fe y arcilla pueden generar la sobre estimación del carbono orgánico. En el caso de la MOS obtenida por el método LOI a $600{ }^{\circ} \mathrm{C}$, se registraron valores elevados respecto al resto de tratamientos LOI, resultado que se relaciona con la pérdida de agua estructural de la arcilla.
La ecuación generada del método LOI a $430{ }^{\circ} \mathrm{C}$ durante dos horas para predecir COS es el más adecuado en suelos cultivados con cacao de origen aluvial (órdenes: Inceptisol y Alfisol) en la provincia de El Oro, como alternativa si no se dispone de un analizador elemental o en sustitución del método WB. El trabajo demostró que el método LOI a $430{ }^{\circ} \mathrm{C}$ tiene potencial para ser utilizada como una técnica para estimar la MOS; pero su mayor limitación que presenta este tipo de calibración por ecuación lineal es que su aplicación es de carácter local, debe ajustarse para un rango de suelos lo más homogéneo posible, dado que las variaciones de los componentes del suelo son los generadores de la alta variabilidad que puede llegar a obtenerse.

\section{AGRADECIMIENTOS}

Este trabajo fue apoyado por la técnica de la Universidad de Machala, bajo la subvención HCU 503-2017. 


\section{BIBLIOGRAFÍA}

Alves Fernandes RB, de Carvalho Junior I A, Silva Ribeiro Junior E, de Sá Mendonça E. 2015. Comparison of different methods for the determination of total organic carbon and humic substances in Brazilian soils. Revista Ceres, 62(5): 496-501. https://doi. org/10.1590/0034-737X201562050011

Barrezueta-Unda S. 2019. Properties of several soils cultivated with cocoa in the province of El Oro, Ecuador. CienciaUAT, 14(1): 155-166. https://doi.org/doi.org/10.29059/ cienciauat.v14i1.1210

Bianchi SR, Miyazawa M, Oliveira EL de, Pavan MA. 2008. Relationship between the mass of organic matter and carbon in soil. Brazilian Archives of Biology and Technology, 51(2): 263-269. https://doi.org/10.1590/ S1516-89132008000200005

Boyle J. 2004. A comparison of two methods for estimating the organic matter content of sediments. Journal of Paleolimnology, 31(1): 125-127. https://doi.org/10.1023/B:JOPL.0000013354.67645.df

Dias RD, De Abreu CA, De Abreu MF, Paz-Ferreiro J, Matsura EE, Gonzalez, AP. 2013. Comparison of Methods to Quantify Organic Carbon in Soil Samples from São Paulo State, Brazil. Communications in Soil Science and Plant Analysis, 44(1-4): 429-439. https://doi.org/Doi 10.1080/00103624.2013.742345

Díaz Zorita M. 1999. Soil organic carbon recovery by the Walkley Black method in a typic hapludoll. Communications in Soil Science and Plant Analysis, 30(5-6): 739-745. https://doi.org/10.1080/00103629909370242

Drover DP, Manner H. 1975. A comparison between the walkley black and a dry combustion method for determining organic car- bon in a humic brown soil, Papua New Guinea. Communications in Soil Science and Plant Analysis, 6(5): 495-500. https://doi. org/10.1080/00103627509366586

Fassbender H, Bornemisza E. 1987. Química de suelos: con énfasis en suelos de América Latina (II). IICA.

Gelati P, Sarli G, Germán C, Lozano L, Filgueira R. 2009. Influencia de la materia orgánica en la superficie específica de un Argiudol típico. Revista de La Facultad de Ciencias Agrarias Universidad Del Cuyo, 41(2): 117-126.

Hartemink AE. 2005. Nutrient stocks, nutrient cycling, and soil changes in cocoa ecosystems: A review. In World Soil Information (pp. 227-253). Advances in Agronomy.

Hoogsteen MJJ, Lantinga EA, Bakker EJ, Groot JCJ, Tittonell PA. 2015. Estimating soil organic carbon through loss on ignition: effects of ignition conditions and structural water loss. European Journal of Soil Science, 66(2): 320-328. https://doi.org/10.1111/ejss.12224

Hoogsteen MJJ, Lantinga EA, Bakker EJ, Tittonell PA. 2018. An Evaluation of the Loss-on-Ignition Method for Determining the Soil Organic Matter Content of Calcareous Soils. Communications in Soil Science and Plant Analysis, 49(13): 1541-1552. https://doi.org/10.1080/00103624.2018.147 4475

Howard PJ, Howard DM. 1990. Use of organic carbon and loss-on-ignition to estimate soil organic matter in different soil types and horizons. Biology and Fertility of Soils, 9, 306-310.

Kamara A, Rhodes ER, Sawyerr PA. 2007. Dry Combustion Carbon, Walkley-Black Carbon, and Loss on Ignition for Aggregate Size Fractions on a Toposequence. Communications in Soil Science and Plant Analysis, 38(15-16): 2005-2012. https://doi. org/10.1080/00103620701548639 
Maranhão DDC, Pereira MG, Fontana A, Vital VF, Valladares GS. 2017. Comparison of methods for determination of carbon in calcareous soils. Communications in Soil Science and Plant Analysis: (48) 1646-1658. https:// doi.org/10.1080/00103624.2017.1373797

Martínez JM, Galantini JA, Duval ME, López, FM, Iglesias JO. 2018. Estimating soil organic carbon in Mollisols and its particle-size fractions by loss-on-ignition in the semiarid and semihumid Argentinean Pampas. Geoderma Regional, 12(December 2017), 49-55. https://doi.org/10.1016/j.geodrs.2017.12.004

Mikhailova EA, Noble RRP, Post CJ. 2003. Comparison of Soil Organic Carbon Recovery by Walkley-Black and Dry Combustion Methods in the Russian Chernozem. Communications in Soil Science and Plant Analysis, 34(13-14): 1853-1860. https://doi. org/10.1081/CSS-120023220

Moreno J, Sevillano G, Valverde O, Loayza V. Haro R, Zambrano J. 2016. Soil from the Coastal Plane. In J. Espinosa, J. Moreno, \& G. Bernal (Eds.), The Soils of Ecuador (pp. 1-195). Springer International Publishing. https://doi.org/10.1007/978-3-319-20541-0

Morona F, Melquíades FL, Muller, MM L. 2017. Quantification of Organic Matter in Agricultural Soils from the Central Region of Paraná State, Brazil. Communications in Soil Science and Plant Analysis, 48(19): 2288-2293. https://doi.org/10.1080/00103624.2017.141 1505.Pérez-Flores J, Pérez AA, Suárez YP, Bolaina VC, Quiroga, AL 2017. Leaf litter and its nutrient contribution in the cacao agroforestry system. Agroforestry Systems, 92(2): 365-374. https://doi.org/10.1007/ s10457-017-0096-3

Pribyl DW. 2010. A critical review of the conventional SOC to SOM conversion factor. Geoderma, 156(3-4): 75-83. https://doi.org/10.1016/j.geoderma.2010.02.003
Richardson JL, Bigler RJ.1982. Comparison of walkley black and dry combustion organic carbon determinations in calcareous water logged North Dakota soils. Communications in Soil Science and Plant Analysis, 13(3): 175-183. https://doi. org/10.1080/00103628209367256

Salehi MH, Hashemi-Beni O, Beigi-Harchegani $\mathrm{H}$, Esfandiarpour-Borujeni I, Motaghian $\mathrm{H}$. 2011. Refining Soil Organic Matter Determination by Loss-on-Ignition. Pedosphere, 21(4): 473-482.

Sato JH, Figueiredo CC de, Marchão RL, Madari BE, Benedito LEC, Busato JG, Souza, DM de. 2014. Methods of soil organic carbon determination in Brazilian savannah soi1s. Scientia Agricola, 71(4): 302-308. https:// doi.org/10.1590/0103-9016-2013-0306

Schulte EE. 1987. Recommended soil organic matter test. In J. Peters (Ed.), Wisconsin Procedures for Soil Testing, Plant Analysis and Feed \& Forage Analysis (pp. 52-60). University of Wisconsin. http:// citeseerx.ist.psu.edu/viewdoc/download?doi=10.1.1.164.3981\&rep=rep1\&type $=$ pdf

Schulte EE., Hopkins BG. 1996. Estimation of soil organic matter by weight loss-on-ignition. In F. R. Magdoff, M. A. Tabatabai, \& E. A. Hanlon (Eds.), Soil organic matter: Analysis and interpretation (pp. 21-30). SSSA.

Snoeck D. Koko L, Joffre J, Bastide P, Jagoret P. 2016. Cacao nutrition and fertilization. In E. Lichtfouse (Ed.), Sustainable Agriculture Reviews (Vol. 19, pp. 1-34). Springer International Publishing. https://doi. org/10.1007/978-3-319-26777-7

SPSS, I. 2013. SPSS Statistics for Windows (No. 21). IBM Corp. https://www.ibm.com/us-en/ marketplace/spss-predictive-analytics-enterprise. 
van Vliet J, Slingerland M, Giller K. 2015. Wang J, Wang X, Zhang J. 2013. Evaluating Mineral Nutrition of Cocoa. Wageningen University and Research Centre. https://doi. org/10.1016/bs.agron.2016.10.017

Vitti C, Stellacci, AM, Leogrande R, Mastrangelo M, Cazzato E, Ventrella D. 2016. Assessment of organic carbon in soils: a comparison between the Springer-Klee wet digestion and the dry combustion methods in Mediterranean soils (Southern Italy). CATENA, 137: 113-119. https://doi.org/10.1016/j.catena.2015.09.001

Walkley A, Black A. 1934. An examination of the determination method for determining soil organic matter and a proposed modification of the chromic acid titration method. Soil Science, 37, 29-38.

Wang X, Wang J, Zhang J. 2012. Comparisons of Three Methods for Organic and Inorganic Carbon in Calcareous Soils of Northwestern China. PLoS ONE, 7(8): e44334. https://doi. org/10.1371/journal.pone.0044334

Loss-on-Ignition Method for Determinations of Soil Organic and Inorganic Carbon in Arid Soils of Northwestern China. Pedosphere, 23(5): 593-599. https://doi.org/10.1016/ S1002-0160(13)60052-1

Westman CJ, Hytönen J, Wall A. 2006. Loss on Ignition in the Determination of Pools of Organic Carbon in Soils of Forests and Afforested Arable Fields. Communications in Soil Science and Plant Analysis, 37(7-8): 1059-1075. https://doi. org/10.1080/00103620600586292

Zhang M, Li Y, Stoffella PJ. 2005. Comparison of Analytical Methods for Organic Matter in Composts and Organic Mulches. Communications in Soil Science and Plant Analysis, 36 (17-18): 2587-2599. https://doi. org/10.1080/00103620500257317 